FACULDADE DE CIÊNCIAS ECONÔMICAS DA UFRGS Análise
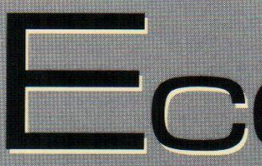

VALORES E PREÇOS DE PRODUÇÃO: UMA RELETURA de MARX Alfredo SAAD FILHO

OS ANTIGOS, OS NOVOS E OS NEO-INSTITUCIONALISTAS: HÁ CONVERGÉ NCIA TEÓ RICA NO PENSAMENTO INSTITUCIONALISTA

Octavio Augusto C. Conceição

A MENSURAÇÃ O DO GRAU DE INDEPENDÉNCIA DO BANCO CENTRAL: UMA ANÁLISE DE SUAS FRAGILIDADES HeLder Ferreira de MENDONÇA

A SUSTENTABILIDADE DA DÍVIDA MOBILIÁ RIA FEDERAL BRASILEIRA: UMA INVESTIGAÇĀ O ADICIONAL VIVIANE LUPORINI

O MODELO dE KRUGMAN EXPLICA A CRISE CAMBIAL. BRASILEIRA EM JANEIRO DE 1999?

Adriano CAMPOS MENEZES E TITO BELCHIOR S. MOREIR/

ESTRANGULAMENTO DA díVIDA EXTERNA E CRESCIMENTO ECONO MICO NA AMÉRICA LATINA: LIÇÓ ES DA DÉCADA DE 80

Marco Vaies buratto E SABino da SiLva P. JR.

CriaÇÃo e desvio de COMÉrCio: ANÁlise do FLuXo COMERCial ENTRE O Brasil. e O MERCOSUl para Alguns PRODUTOS INDUSTRIALIZADOS

Clá Udio Roberto fó fFano Vasconcelos

A EVOLUCÁ̃ DA INDÚ STRIA BRASILEIRA DE CELULOSE E SUA ATUAÇÃO NO MERCADO MUNDIAL

Emerson Martins HILGemberg e Carlos josé Caetano BACHA

A PROTEÇÃo SOCIAL NO PRIMEIRO QUARTEL DO SÉCULO XXI: BRINCANDO COM CENÁRIOS

rosa Maria Marques e Áquilas Mendes

ECONOMIA DO CRIME: ELEMENTOS TEÓ RICOS E EVIDÊNCIAS EMPÍRICAS

Gilberto José SChaefer e Pery Francisco Assis Shikida

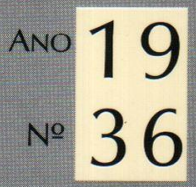


UNIVERSIDADE FEDERAL DO RIO GRANDE DO SUL

Reitora: Profa. Wrana Maria Panizzi

FACULDADE DE CIÊNCIAS ECONÔMICAS

Diretor: Prof. Pedro Cézar Dutra Fonseca

CENTRO DE ESTUDOS E PESQUISAS ECONÔMICAS

Diretor: Prof. Gentil Corazza

DEPARTAMENTO DE CIÊNCIAS ECONÔMICAS

Chefe: Prof. Luiz Alberto Oliveira Ribeiro de Miranda

DAPARTAMENTO DE CIÊNCIAS CONTÁBEIS E ATUARIAIS

Chefe: João Marcos Leão da Rocha

CURSO DE PÓS-GRADUAÇÃO EM ECONOMIA

Coordenador: Prof. Eduardo Pontual Ribeiro

CURSO DE PÓS-GRADUAÇÃO EM ECONOMIA RURAL

Coordenador: Prof. Jalcione Almeida

CONSELHO EDITORIAL: Achyles B. Costa, Aray M. Feldens, Carlos A. Crusius, Carlos G. A. Mielitz Netto, Eduardo A. Maldonado Filho, Eduardo P. Ribeiro, Eugênio Lagemann, Fernando Ferrari Filho, Gentil Corazza, Jan A. Kregel (Univ. of Bologna), Marcelo S. Portugal, Nali J. Souza, Otilia B. K. Carrion, Paulo A. Spohr, Paulo D. Waquil, Pedro C. D. Fonseca, Philip Arestis (Univ of East London), Roberto C. Moraes, Ronald Otto Hillbrecht, Stefano Florissi, Eleutério F. S. Prado (USP), Fernando H. Barbosa (FGV/RJ), Gustavo Franco (PUC/RJ), João R. Sanson (UFSC), Joaquim P. Andrade (UnB), Juan H. Moldau (USP), Paul Davidson (Univ. of Tennessee), Werner Baer (Univ. of Illinois).

COMISSÃO EDITORIAL: Eduardo Augusto Maldonado Filho, Fermando Ferrari Filho, Gentil Corazza, Marcelo Savino Portugal, Paulo Dabdab Waquil; Roberto Camps Moraes.

EDITOR: Fernando Ferrari Filho

EDITOR ADJUNTO: Pedro Silveira Bandeira

SECRETÁRIA: Vanessa Hoffmann de Quadros

REVISÃO DE TEXTOS: Vanete Ricacheski

FUNDADOR: Prof. Antônjo Carlos Santos Rosa

Os materiais publicados na revista Análise Econômica sāo da exclusiva responsabilidade dos autores. É permitida a reproduçāo total ou parcial dos trabalhos, desde que seja citada a fonte. Aceita-se permuta com revistas congêneres. Aceitam-se, também, livros para divulgação, elaboração de resenhas e recensões. Toda correspondência, material para publicação (vide normas na terceira capa), assinaturas e permutas devem ser dirigidos ao seguinte destinatário:

PROF. FERNANDO FERRARI FILHO

Revista Análise Econômica - Av. João Pessoa, 52 CEP 90040-000 PORTO ALEGRE - RS, BRASIL Telefones: (051) 316-3348 e 316-3440 - Fax: (051) 316-3990

E-mail: rae@vortex.ufrgs.br

Análise Econômica

Ano 18, n⿳3 33, março, 2000 - Porto Alegre

Faculdade de Ciências Econômicas, UFRGS, 2000

Periodicidade semestral, março e setembro.

1. Teoria Econômica - Desenvolvimento Regional -

Economia Agrícola - Pesquisa Teórica e Aplicada -

Periódicos. I. Brasil

Faculdade de Ciências Econômicas,

Universidade Federal do Rio Grande do Sul.

CDD 330.05

CDU 33 (81) (05) 


\title{
O modelo de Krugman explica a crise cambial brasileira em janeiro de 1999?"
}

\author{
Adriano Campos Menezes ${ }^{* *}$ e Tito Belchior S. Moreira ${ }^{* * *}$
}

\begin{abstract}
Resumo: Krugman (1979) desenvolveu um modelo canônico de crises cambiais e ataques especulativos que deu origem aos chamados "modelos de $1^{\text {a }}$ geração". Nesse contexto, esse trabalho tem por objetivo a análise do modelo de Krugman (1979) para o Brasil, no periodo de janeiro de 1995 à dezembro de 1998. Para isso, utilizamos o teste de causalidade de Granger (1969) que testa a precedência temporal entre reservas internacionais e crédito interno. Os resultados revelam o caso de independência, tendo em vista que não ocorreu causalidade unidirecional, tampouco bidirecional ou simultaneidade. Portanto, concluímos pela insustentabilidade da proposição de Krugman (1979).
\end{abstract}

Palavras-chave: Causalidade de Granger; ataque especulativo; crise cambial.

\begin{abstract}
Krugman (1979) developed a canonical model of currency crises and speculative attack. Later, it was called "models of 1 st generation ". In this context, this paper analyzes the model of Krugman (1979) to Brazil, in the period of January of 1995 to December of 1998. We used the test of Granger Causality to test the temporary precedence between international reserves and domestic credit. The results reveal the case of independence causality, since it didn't happen unidirectional causality, bi-directional causality or simultaneity causality. Therefore, the proposition of Krugman (1979) is untenable.
\end{abstract}

Key words: Granger causality; speculative attack; currency crises.

\footnotetext{
- Os autores agredecem a Adolfo Sachsida e aos pareceristas pelos pertinentes comentários. Como de praxe, a resposibilidade pelos erros e omissões deste artigo cabe exclusivamente aos autores. Destaque-se que este trabalho é resultante de um projeto na área de concentraçāo de finanças internacionais da Universidade Católica de Brasilia.

“Mestrando pela Universidade Católica de Brasília - UCB; e-mail: dricomenezes@bol com.br e menezesadriano(@aol.com.

".. Professor do Departamento de Economia da Universidade Católica de Brasilia - UCB; e-mail: tito@ucb.br e titoeco@uol.com.br.
} 


\section{Introdução}

Um marco na literatura econômica a respeito dos ataques especulativos teve início com Krugman (1979). O autor desenvolveu um modelo clássico. Se o governo, para financiar o déficit em suas contas, emite mais moeda do que o setor privado está disposto a sustentar, isto implica, sob um regime de câmbio fixo, um declínio gradual das reservas internacionais. Este fato, implica o abandono por parte do Banco Central do regime de câmbio fixo, culminando com a desvalorização da moeda nacional e a aceitação da flexibilidade do regime cambial.

Destarte, as proposições de Krugman (1979) implicam uma relação de causalidade entre as reservas internacionais e o crédito interno. O governo somente poderia financiar o seu déficit, na medida em que promovesse uma expansão monetária, que não ocorreria sem alavancagem do crédito interno e sem um gradual declínio das reservas internacionais.

Objetiva-se, nesse trabalho, testar o modelo de Krugman (1979) para o Brasil, no período de janeiro de 1995 a dezembro de 1998. Para isso, utilizamos o teste de causalidade de Granger (1969) que testa a precedência temporal entre reservas internacionais e crédito interno. Na seção 2 faremos uma breve discussão sobre crises cambiais e na próxima descrevemos o modelo de Krugman (1979). Na seção 4, analisamos os resultados. A última parte conclui as questões levantadas nesse trabalho.

\section{Crises cambiais}

Um marco para a literatura econômica sobre ataques especulativos e crises cambiais tem início com o trabalho de Krugman (1979). Segundo sua conceituação, uma crise padrão ocorre da seguinte maneira: admita um país que adote um regime de câmbio fixo, assumindo que a fixação dá-se mediante a intervenção da autoridade monetária no mercado cambial. Nessas condições, se o governo, concomitantemente, com vistas a financiar o déficit de suas contas, emite mais moeda do que o setor privado está disposto a sustentar, os investidores, dada uma taxa de câmbio fixa, trocarão a moeda nacional em excesso em suas carteiras por moeda estrangeira. A essa paridade cambial, as reservas internacionais do governo gradualmente declinam. 
Como resultado, ocorre o ataque especulativo que torna o governo incapaz de defender-se, forçando-o a abandonar o regime de câmbio fixo.

$\mathrm{O}$ ataque especulativo contra as reservas do governo pode ser visto como um processo em que os investidores alteram a composição de suas carteiras, reduzindo a proporção de ativos domésticos e aumentando a proporção de ativos em moeda estrangeira. Esta alteração na composição é então justificada como uma forma de defesa pelos investidores, quando o governo mostra-se incapaz de defender a taxa de câmbio, levando à depreciação do preço da moeda nacional. A principal conclusão é que, se os investidores corretamente antecipam os eventos, as crises são um resultado natural da maximização do comportamento dos investidores.

Neste primeiro trabalho, o próprio Krugman admitiu duas limitações que envolviam suas análises. A primeira refere-se ao seu modelo como um ato de simplificação macroeconômica. A segunda, assume que o modelo não revelava todos os possíveis meios de intervenção do governo, já destacando a importância da incorporação de outras políticas para estabilização do mercado de câmbio, tais como as operações no mercado aberto ou mesmo a intervenção no mercado de futuros. O banco central possui uma postura passiva diante da possibilidade de um ataque especulativo.

Krugman (1979) explicou a lógica do ataque especulativo, mas não derivou uma equação de probabilidade de ocorrência de crises cambiais e ataques especulativos. Esses objetivos foram atingidos posteriormente com o trabalho de Flood e Garber (1984). Os autores transformaram o modelo anterior em linear e estocástico. Nesses modelos, a expansão constante do crédito doméstico líquido, derivado de um desequilibrio fiscal e financiado pela autoridade monetária, leva a um declínio das reservas. Essa família de modelos é conhecida - na literatura de crises cambiais como modelos de primeira geração. Essas crises, segundo essa corrente, provêm de inconsistências nos fundamentos, que, de forma geral, têm origem nos desajustes entre as políticas monetária, fiscal e a cambial.

No entanto, como observou Schwartsman (1999), apesar de estes modelos serem uma descrição acurada de crises, o "modelo canônico não parecia, contudo, capaz de explicar" a ocorrência da crise do Sistema Monetário Europeu em 1992, em particular a espetacular quebra da libra face ao ataque desferido por George Soros. Nesses países, os ataques especulativos não decorreram de problemas nos fundamentos.

Por esta razão, para solucionar uma visível contradição da formulação básica acerca dos pressupostos teóricos na literatura de ataques especulativos, surgiram os chamados modelos de segunda geração. Como 
destacaram Schwartsman (1999) e Miranda (1999), a reconciliação entre os fatos e a teoria foi restabelecida através do trabalho de Obstfeld (1994), que desenvolveu um modelo de equilíbrio múltiplo. Esta modelagem indica que a autoridade monetária é colocada diante de duas opções contraditórias: i) caso a prioridade seja a defesa da estabilidade incorporada na taxa de câmbio fixa, o Banco Central pode expandir a dívida externa, em antecipação à exaustão das reservas internacionais, evitando a crise anunciada através das especulações no mercado de ativos; ii) caso o custo de defesa (recessão) da taxa de câmbio seja maior que o ganho da estabilidade (reputação), a opção do governo será o abandono da fixação cambial. Cabe destacar que o enfoque nos modelos desenvolvidos a partir desta análise explicam que as crises não procedem de problemas nos fundamentos econômicos, mas sim de uma profecia auto-realizável. "A diferença fundamental consiste na expectativa que os agentes privados têm de que a política econômica adotada pelo governo é exógena (fundamentos) ou endógena (equilíbrio múltiplo) à ocorrência de ataques especulativos" (Miranda, 1999, p. 26).

Nas décadas 80 e 90, vários países em desenvolvimento - especialmente aqueles que se recuperaram da crise da dívida nos anos 80, entre eles, México, Argentina e Brasil - passaram por experiências de estabilização ancorados ao câmbio. No Brasil, em relação aos efeitos do programa de estabilização que teve início em 1994, e sua flexibilização ocorrida em Janeiro de 1999, Mollo e Silva (1999) analisaram e discutiram em torno dos efeitos monetários sobre a economia. Elas concluíram quanto à manutenção da âncora cambial: se, por um lado, mostrou-se eficiente para solucionar o vilão da inflação; por outro, mostrou-se suficientemente capaz de aumentar o custo social, deteriorar a situação financeira e imobilizar o poder monetário do Estado. Em conformidade com essas afirmativas, mas aproximando-se do debate dos modelos de primeira e segunda geração de ataques especulativos, Miranda (1999) e Schwartsman (1999) chegam a uma conclusão: a recente crise cambial brasileira deve-se a uma mistura entre os desajustes nos "fundamentos" macroeconômicos, baseados no modelo original de Krugman, e a atuação de algum mecanismo de "contágio", como afirma Obstfeld (1994). Já para Hermann (1999), a explicação tanto para a crise brasileira de 1997 quanto para a de 1998 pode ser definida como desprovida de expectativas racionais sobre os "fundamentos", mas adequadamente descritas como "crises de contágio". 
Assim, tendo em vista os objetivos propostos por este trabalho quanto ao entendimento da crise cambial brasileira, definidos anteriormente, na próxima seção desenvolve-se o modelo de Krugman, bem como suas conclusões face às ocorrências de ataques especulativos, na medida em que persistem desajustes nos fundamentos macroeconômicos.

\section{Modelo de crise do balanço de pagamento de Krugman (1979)}

Originalmente, admiti-se uma pequena economia na qual os residentes de um país consomem apenas um bem comercializável. A oferta interna deste bem é exógena, sendo o seu preço internacional fixo e igual a um. O nível de preço interno é igual a taxa de câmbio nominal, dada a condição da paridade do poder de compra (PPP). Os agentes detêm três categorias de ativos: moeda nacional, e títulos domésticos e externos, que são perfeitos substitutos. Não há bancos privados. O estoque de moeda é determinado pela soma do total de crédito doméstico emitido pelo banco central com as reservas internacionais, em valores correntes da moeda nacional, na qual é mantida pelo Banco Central. Finalmente, os agentes têm informações perfeitas sobre o mercado. $\mathrm{O}$ modelo é definido através do ajuste das seguintes equações:

$$
\begin{array}{lr}
m_{t}-p_{t}=\overline{\phi y}-\alpha i_{t} & \phi, \alpha>0 \\
m_{t}=\gamma D_{t}+(1-\gamma) R_{t} & 0<y<1 \\
\dot{D}_{t}=\mu & \mu>0 \\
p_{t}=e_{t} & \\
i_{t}=i^{*}+\dot{e}_{t} &
\end{array}
$$

Todas as variáveis são mensuradas em logaritmos, exceto as taxas de juros. Assim, temos que:

$$
\begin{aligned}
& m_{t}=\text { Estoque nominal de moeda } \\
& D_{t}=\text { Crédito doméstico } \\
& R_{t}=\text { Reservas internacionais em valor doméstico corrente } \\
& e_{t}=\text { Taxa de câmbio à vista } \\
& p_{t}=\text { Nível de preços } \\
& \bar{y}=\text { Produto (variável exógena) }
\end{aligned}
$$


$i^{*}=$ Taxa de juros internacional (constante)

$i_{t}=$ Taxa de juros interna

Primeiramente, verifica-se que na equação 1.1 a demanda por moeda é função positiva da renda e negativa da taxa de juros doméstica. A equação I.2 é uma aproximação log-linear da identidade, que define o estoque de moeda como a soma das reservas e do crédito doméstico, o qual cresce a uma taxa $\mu$ (equação 1.3). Em seguida, as equações $1.4 \mathrm{e}$ 1.5 definem, respectivamente, a paridade do poder de compra e a paridade descoberta.

Ajustando $\delta=\phi \bar{y}-\alpha i^{*}$ e combinando as equações $1.1,1.4$ e 1.5, tem-se que,

$$
m_{t}-e_{t}=\delta-\alpha \dot{e_{t}}
$$

Tendo em vista que a taxa de câmbio nominal é fixa, tem-se que $e_{t}=\bar{e}$ e $e_{t}=0$, logo, simplifica-se a última equação.

$$
m_{t}-\bar{e}_{t}=\delta
$$

Desta forma, mostra-se que o banco central acomoda as variações na demanda por moeda doméstica através da venda ou compra de reservas internacionais para o público. Sendo assim, utilizando as equações 1.2 e 1.6', tem-se a seguinte relação,

$$
R_{t}=\frac{\delta+\bar{e}-\gamma D_{t}}{1-\gamma} \quad \gamma>0
$$

E, usando a equação 1.3, tem-se:

$$
\dot{R}_{t}=-\frac{\mu}{\theta}, \quad \theta \equiv \frac{1-\gamma}{\gamma}
$$

Por último, a equação 1.8 indica que, se o crédito doméstico expandir excessivamente, as reservas cairiam proporcionalmente à taxa de expansão do crédito. Por esta razão, um estoque limitado de reservas internacionais será exaurido em um determinado período de tempo, obedecidas estas condições. 


\section{Resultados}

A metodologia relativa aos resultados apresentados nessa seção, sobre testes de causalidade entre crédito doméstico e reservas internacionais, é apresentada em Carneiro (1997), Charemza e Deadman (1997), Divino (1998) e Granger (1969).

\subsection{Modelagem das variáveis}

Neste estudo são analisadas as variáveis Reservas Internacionais e o Crédito Interno Líquido, utilizando-se informações mensais para o período de janeiro de 1995 a dezembro de 1998. Estas séries foram dispostas em uma função do tipo log-linear. Quanto à origem, as Reservas Internacionais foram obtidas mediante consulta a boletins do Banco Central (BACEN). Os valores desta série estavam disponibilizados em US\$ milhões, agregando o conceito de liquidez do BACEN. Posteriormente, estes dados são convertidos para Reais, multiplicando-se pela Taxa de Câmbio(venda) de R $\$ / \mathrm{US} \$$, também obtida via Boletins do BACEN. O Crédito Interno Líquido também tem como fonte primária o BACEN, medido em $\mathrm{R} \$$ milhões, apesar de ter sido coletado junto ao Fundo Monetário Internacional, International Financial Statistics(1999).

Para facilitar o entendimento, adotamos as siglas RES e CRE, que significam, respectivamente, Reservas Internacionais e o Crédito Interno Líquido.

\subsection{Testando a hipótese de raiz unitária}

Inicialmente, testou-se a ordem de integração das variáveis. Como o termo aleatório segue um ARMA (p,q), utilizou-se o teste Dickey-Fuller aumentado (augmented Dickey-Fuller - ADF), feito com 13 defasagens, de acordo com a sugestão de Doornik e Hendry (1994), para dados mensais. O teste foi efetuado para cada variável, sendo a permanência do intercepto (constante) e a tendência (tend) condicionados à significância, ou não, destes componentes determinísticos. Quanto à escolha do número de defasagens para o teste ADF, seguiu-se a metodologia de Doornik e Hendry (1994). O teste equivale ao de DickeyFuller (1979) simples - a defasagem do ADF iguala-se a zero.

Os resultados dos testes de raiz unitária são apresentados na tabela 1 , discriminando as variáveis, e respectivamente, os valores calculados 
para o teste $\mathrm{ADF}$, bem como as defasagens consideradas relevantes. Neste trabalho, utilizou-se, tanto para o teste de raiz unitária como para as análise de cointegração, as estatísticas de Mackinnon (1991). Como destaca Alencar (1998), "a vantagem da utilização destas estatísticas é que elas apresentam um resultado específico para o número de variáveis que estão sendo utilizadas na equação de cointegração, além de mostrar os valores críticos para qualquer tamanho de amostra, assim como um valor específico quando da inclusão, ou não, da constante e da tendência".

Tabela 1: Testes de raíz unitária

\begin{tabular}{l|c|c|c|c}
\hline Variável & ADF & Lag $^{(1)}$ & Comp. Det. ${ }^{(2)}$ & Resultado \\
\hline In(RES) & $-2,383$ & 4 & tend $^{(3)}$, constante & $\mathrm{I}(1)$ \\
$\ln (\mathrm{CRE})$ & $-0,9902$ & 0 & tend, constante & $\mathrm{I}(1)$ \\
$\operatorname{Dln}(\mathrm{RES})$ & $-4,877 * *$ & 4 & tend, constante & $\mathrm{I}(0)$ \\
Dln(CRE) & $-9,998^{* *}$ & 0 & tend, constante & $\mathrm{I}(0)$ \\
\hline Obs.: ${ }^{*}$ - significa que o teste regeita a hipótese nula de rá́z unitária ao de significância de $5 \%, \mathrm{e}^{* *}$ \\
de 1\%. \\
1) Lag é a defasagem considerada no ADF; \\
2) Comp. Det. - componente determinístico utilizado no teste; \\
3) tend. - tendência; \\
D significa variável em diferença e In, logaritmo neperiano.
\end{tabular}

Assim, a observação dos resultados expostos na tabela 1, suficientemente, demonstram que as variáveis em nível são todas I(1), ou seja, não rejeitam a hipótese de raiz unitária. Como a maioria das séries econômicas, os testes de raiz unitária mostram que primeira diferença dessas variáveis são I(0), isto é, são estacionárias. Não há registro de quebras estruturais no período estudado - o teste Chow não indica mudança na constância dos parâmetros estimados.

\subsection{Estimando a equação de longo prazo}

Neste caso, uma vez que as variáveis não são estacionárias em nível, foi estimada uma regressão de cointegração ou equação de longo prazo, equação 1, para saber se há um relacionamento de equilíbrio entre estas variáveis não estacionárias, no longo prazo. Os resultados são apresentados na tabela 2, conjuntamente com o teste de cointegração de Engle e Granger (1987). Neste estudo, opta-se por este 
método, tendo em vista que, para o teste de causalidade proposto, busca-se somente a existência de um único vetor de cointegração. Em análises de cointegração de sistemas, Madala e Kim (2000) e Alencar (1998) sugerem a metodologia de Johansen (1988), a qual permite testar e estimar múltiplos vetores de cointegração, uma alternativa além do âmbito deste trabalho. No entanto, Carneiro (1997) afirma que tanto o método de cointegração por Engle e Granger (1987) como o mais recente proposto por Johansen (1988) são válidos para esta etapa.

$$
\ln \left(\operatorname{RES}_{\mathrm{t}}\right)=\mathrm{a}_{0} \ln \left(\mathrm{CRE}_{\mathrm{t}}\right)+\mu_{t}
$$

Tabela 2: Estimação de $\ln (\mathrm{RES})$ por $\mathrm{MQO}$

Período amostral: 1995 (1) - 1998 (12)

\begin{tabular}{l|c|c|c|c}
\hline \multicolumn{1}{c|}{ Variável } & Coeficiente & S.E. $^{(1)}$ & $\boldsymbol{T}^{(2)}$ & HSCE $^{(3)}$ \\
\hline $\ln (\mathrm{CRE}) \mathrm{t}$ & 0,86668 & 0,0022011 & 393,753 & 0,0021997 \\
\hline $\mathrm{R}^{2}=0,999$ & $\mathrm{~S}=0,192$ & $\mathrm{DW}=1,60$ & $\mathrm{RSS}=1,734$
\end{tabular}

\begin{tabular}{l|c|c|c}
\hline & ADF & Lag & Componente Determinístico \\
\hline MCE & $-3,607^{*}$ & 3 & constante \\
MCE & $-2,731$ & 4 & tend, constante \\
\hline
\end{tabular}

Obs.: * "Significativo ao nível de 1\%; * Significativo ao nível de $5 \%$.

1) desvio padrão;

2) estatística t-student;

3) desvio padrāo heterocedástico consciente.

Apesar de as informações estatísticas das variáveis estarem restritas a um pequeno período amostral, as estimativas da tabela 2 permitem inferir que esta equação possui um coeficiente consistente e altamente eficiente, o que, de acordo com os comentários de Perman (1991), é esperado quando existe cointegração. O modelo apresenta um alto coeficiente de determinação $\left(\mathrm{R}^{2}\right)$, bem como um bom desempenho com relação à performance da estatística t-Student, indicando uma probabilidade praticamente nula de rejeitar a hipótese de que variável exógena tenha baixa significância. Com relação aos erros resultantes da estimação pelo método dos Mínimos Quadrados Ordinários (MQO), a comparação entre o desvio padrão (S.E.) e o desvio padrão heteroscedástico consistente (HSCE) não mostra grandes distorções, o que refuta a possível hipótese de que os erros do modelo são heterocedásticos.

O teste de Engle e Granger (1987) para o mecanismo de correção de erros (MCE) foi realizado primeiramente partindo de um teste ADF mais geral. No entanto, a tendência foi excluída por apresentar baixa 
significância, e, em seguida, refazendo-se novamente o teste ADF, e, desta vez, os valores calculados excederam os valores tabelados para o teste, caso em que a hipótese nula de que existe raiz unitária é rejeitada. Portanto, considerou-se que o MCE possui um comportamento estacionário.

Entretanto, por ser o objetivo deste trabalho aplicar um teste de causalidade, deve-se estimar uma segunda equação, para entender um possível equilíbrio inverso entre as variáveis crédito e reservas. A razão disso encontra-se em limitações existentes na metodologia de Engle e Granger (1987). Para um bom entendimento, considere a seguinte explicação. Seguindo o exemplo Ender (1995), para duas variáveis, o teste de Engle e Granger pode ser efetuado por duas direções:

$$
\begin{aligned}
& y_{t}=\gamma_{10}+\gamma_{11} x_{t}+\mu_{1 t} \\
& x_{t}=\gamma_{20}+\gamma_{21} y_{t}+\mu_{2 t}
\end{aligned}
$$

Segundo Alencar (1998), "a teoria assintótica garante que, à medida que a amostra cresce ao infinito, o teste feito $\operatorname{com}\left\{\mu_{k}\right\}$ equivale ao feito com $\left\{\mu_{2 t}\right\}$. Entretanto, não costumamos trabalhar com amostras tão grandes. Sendo assim, é possivel que rejeitemos a hipótese de não cointegração em um dos testes e não no outro. Resultado bastante indesejado". Por esta razão, preferiu-se neste trabalho estimar uma outra equação para verificar se, de alguma forma, existe o caso acima mencionado, já que esse estudo não trabalha com grandes amostras. Os resultados são apresentados na tabela 3 .

Tabela 3: Estimação de In(CRE) por MQO

Período amostral: 1995 (1) - 1998 (12)

\begin{tabular}{l|c|c|c|c}
\hline Variável & Coeficiente & S.E. & $T$ & HS CE \\
\hline $\ln ($ RES)t & 1,1535 & 0,0029295 & 393,753 & 0,002877 \\
\hline $\mathrm{R} 2=0,999$ & $\mathrm{~S}=0,222$ & $\mathrm{DW}=0,223$ & $\mathrm{RSS}=2,308$ &
\end{tabular}

\begin{tabular}{l|c|c|c}
\hline & ADF & Lag & Componente Deterministico \\
\hline MCE & $-3,607^{*}$ & 3 & constante \\
MCE & $-2,732$ & 4 & tend, constante \\
\hline
\end{tabular}

** Significado ao nivel de $1 \%$; ${ }^{*}$ Significado ao nivel de $5 \%$.

Como foi possível visualizar, o coeficiente estimado permanece consistente, enquanto o restante das estimativas não apresenta grandes variaçōes no que diz respeito ao comportamento da primeira equação. $\mathrm{A}$ inversão das variáveis, apesar da existência de um curto período amostral, não trouxe alterações no comportamento do MCE, mantendo-se as mes- 
mas observações que foram enunciadas para o primeiro. Excetua-se apenas um comentário relacionado à estatística de Durbin-Watson (DW), para testar se os erros do modelo são autocorrelacionados. Na primeira estimação, pelo DW nāo é possivel não rejeitar a inexistência de autocorrelação, seja ela positiva ou negativa. De outra forma, a segunda estimativa, ao que parece, possui grandes evidências de uma positiva autocorrelação dos resíduos, tendo em vista que os valores calculados situamse abaixo da zona de indecisão para o referido teste.

\subsection{Estimando a dinâmica de curto prazo}

Após todos os testes empregados anteriormente, os resultados empíricos provam a existência de cointegração entre variáveis do modelo, embora os dados refiram-se a um pequeno período amostral. Sendo assim, seguem-se às análises do comportamento das variáveis, no sentido da causalidade de Granger, no curto prazo.

Com estas perspectivas, estimou-se, primeiramente, a equação 2, a qual conjuntamente fixa os conceitos de cointegração, adicionando ao teste de causalidade o mecanismo de conreção de erros (MCE) ${ }^{1}$.

Equação (2):

$$
\begin{aligned}
D \ln (R E S)= & a_{0}+a_{1} D \ln (R E S)_{t-1}+a_{2} D \ln (R E S)_{t-2}+a_{3} D \ln (R E S)_{t-3} \\
& +a_{4} D \ln (R E S)_{t-4}+a_{5} D \ln (R E S)_{t-5}+a_{6} D(C R E)_{t-1}+a_{7} D(C R E)_{t-2} \\
& +a_{8} D(C R E)_{t-3}+a_{9} D(C R E)_{t-4}+a_{10} D(C R E)_{t-5}+a_{11} D(C R E)_{t-6} \\
& +a_{12} M C E_{t-1}+e_{t}
\end{aligned}
$$

Em síntese, conforme o que foi comentado anteriormente, as variáveis não são estacionárias em nível, necessitando de diferenciação de primeira ordem. Quanto à escolha do número de defasagens, tem-se que: para a variável crédito, considerou-se relevante a inclusão de 6 defasagens; e para as reservas, 5 defasagens, de acordo com os critérios já definidos no capítulo anterior. Assim, os dados referentes à primeira etapa do teste de causalidade encontram-se sumarizados na tabela 4.

A observação dos resultados, ao contrário das relações obtidas com as variáveis em níveis, não indica um alto coeficiente de determinação, e analisando-se o teste F, constata-se que o conjunto dos coeficientes da regressão

\footnotetext{
'Como observa Alencar (1998), "a elaboraçāo de um modelo nas diferenças dessas variáveis que omitisse - MCE seria inapropriada e incorreria num erro de má especificação".
} 
não podem ser considerados diferentes de zero ${ }^{2}$. No que diz respeito aos testes de diagnóstico, a regressão não apresentou uma boa distribuição normal dos resíduos, nem foi capaz de rejeitar a hipótese de uma forma funcional mal especificada, conforme demonstraram o Reset test (Regression Specification Test) e o Normality test, ambos indicados na tabela 4. Todavia, quanto à constância da variância e aos erros de autocorrelação, os testes Autoregressive Conditional Heteroscedasticity (ARCH) e AR 1-4, indicaram, respectivamente, a presença de resíduos homocedásticos e não correlacionados.

Tabela 4: Estimação de Dln(RES) por MQO

Período amostral: 1995 (1) - 1998 (12)

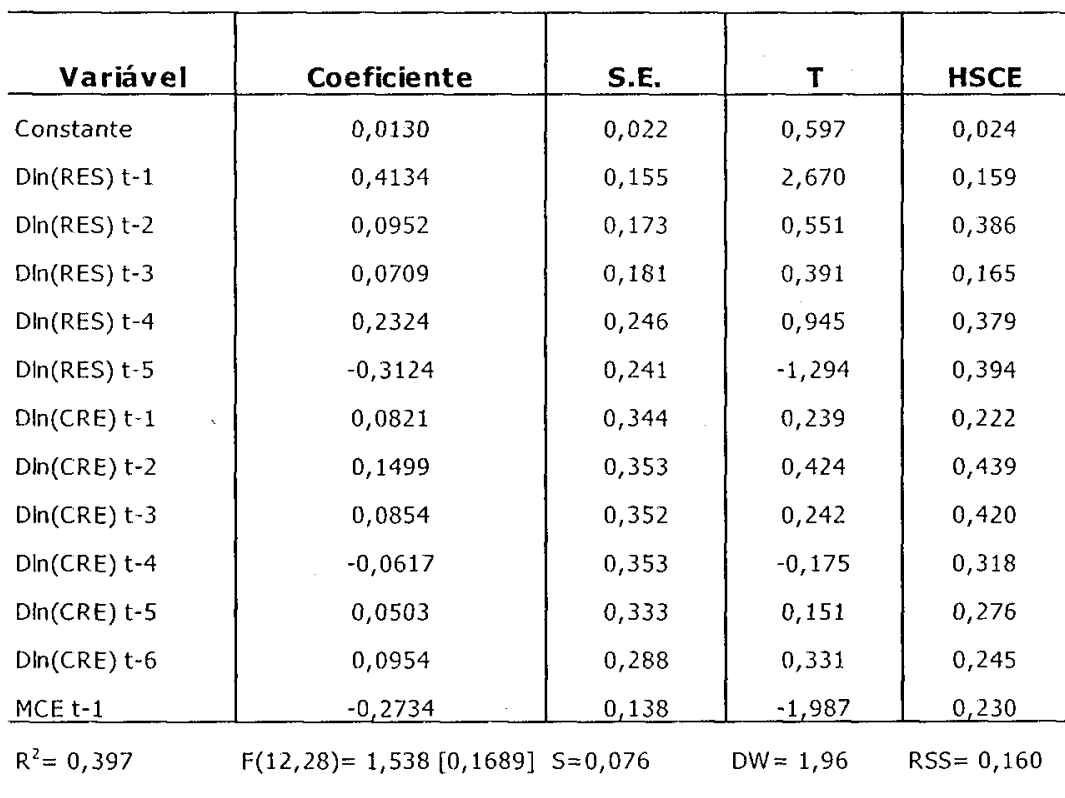

\section{Teste de Causalidade de Granger}

$F(6,28)=0,083034[0,9975]$

\section{Testes de Diagnóstico}

AR 1 - $4 F(4,24)=0,529[0,715]$

ARCH $4 F()=0,049[0,995]$

Normality Chi^2 $(2)=29,428[0,000] * *$

$X^{\wedge} 2 \quad F()=0,077[1,000]$

RESET $F()=8,056[0,008] * *$

* Significativo ao nivel de $1 \%$; "Significativo ao nível de $5 \%$.

${ }^{2}$ Para todos os testes estatísticos, de diagnóstico, e também os testes de causalidade de Granger, considerou-se estatisticamete válidos os resultados sujeitos a intervalo mínimo de $95 \%$ de confiança (nível de $5 \%$ de significância - *). 
Embora sejam importantes estas considerações, cabe considerar especificamente o resultado da significância do conjunto de coeficientes defasados e da significância do coeficiente do termo MCE. Neste intuito, no sentido da causalidade de Granger para a respectiva modelagem, com uma probabilidade de $99 \%^{3}$, o conjunto de coeficientes defasados para a variável explicativa $\operatorname{Din}(\mathrm{CRE})$ não pode ser considerado significativamente diferente de zero. Além disso, fez-se também um teste de significância para o $\mathrm{MCE}^{4}$, obtendo-se o seguinte resultado estatístico: $\mathrm{F}(1,28)=3,9489$ [0,0568], ou seja, com uma probabilidade $6 \%$, o valor defasado do coeficiente do resíduo não pode ser considerado significante. Desta forma, conclui-se pela rejeição da hipótese nula de que Dln(CRE) Granger-causa $\mathrm{D} \ln (\mathrm{RES})$.

Para tornar o teste de causalidade completo, estimou-se ainda a equação 3, que mostra uma modelagem inversa à anterior: Da mesma maneira como foi observado na primeira estimação, os resultados não revelaram-se satisfatórios (ver Tabela 5). Nestas condições, o modelo apresentou um baixo coeficiente de determinação, culminando com um teste $\mathrm{F}$ acusando que o conjunto dos coeficientes não são estatisticamente significantes ${ }^{5}$. Quanto aos testes de diagnóstico, os resultados não apresentaram uma boa distribuição normal dos residuos. Desta vez, ao invés de uma forma funcional mal especificada, a regressão apresenta erros de autocorrelação, como indica o teste AR 1 -4.

$$
\begin{aligned}
\mathrm{D} \ln (\mathrm{CRE})= & \mathrm{a}_{0}+\mathrm{a}_{1} \mathrm{D} \ln (\mathrm{CRE})_{\mathrm{t}-\mathrm{t}}+\mathrm{a}_{1} \mathrm{D} \ln (\mathrm{CRE})_{\mathrm{t}-2}+\mathrm{a}_{2} \mathrm{D} \ln (\mathrm{CRE})_{\mathrm{t}-3} \\
& +\mathrm{a}_{3} \mathrm{D} \ln (\mathrm{CRE})_{\mathrm{t}-4}+\mathrm{a}_{4} \mathrm{D} \ln (\mathrm{CRE})_{\mathrm{t}-5}+\mathrm{a}_{5} \mathrm{D} \ln (\mathrm{CRE})_{\mathrm{t}-6}+\mathrm{a}_{6} \mathrm{D}(\mathrm{RES})_{\mathrm{t}-1} \\
& +\mathrm{a}_{7} \mathrm{D}(\mathrm{RES})_{\mathrm{t}-2}+\mathrm{a}_{8} \mathrm{D}(\mathrm{RES})_{1-3}+\mathrm{a}_{9} \mathrm{D}(\mathrm{RES})_{\mathrm{t}-4}+\mathrm{a}_{10} \mathrm{D}(\mathrm{RES})_{\mathrm{t}-5} \\
& +\mathrm{a}_{12} \mathrm{MCE}_{\mathrm{t}-1}+\mathrm{e}_{1}
\end{aligned}
$$

O teste de causalidade também não apresenta novidades. O teste F para significância do conjunto dos coeficientes defasados da variável Dln(RES), ao nivel de 5\% de significância, mostrou-se, estatisticamente

\footnotetext{
${ }^{3}$ Os valores entre [ ] indicam a probabilidade do valor F sob a hipótese nula para uma distribuição $\mathrm{F}$, de acordo com os graus de liberdade indicados. Neste trabalho, os testes estão dispostos segundo a notação do PcGive.

${ }^{4}$ Segundo Galrāo (1999), "a intuição é de que se as duas variáveis forem cointegradas, então parte da mudança corrente em $\mathrm{X}$ pode ser o resultado de movimentos corretivos em $\mathrm{Y}$ para que se atinja novamente o equilíbrio de longo prazo com a variável $X$ Desde que $X$ e $Y$ possuam um tendência em comum, a causalidade deverá existir pelo menos em um direção".

${ }^{5}\left[\mathrm{~F}_{0.5}(12,28)=2,12\right]$ representa o valor tabelado para um intervalo de $95 \%$ de confiança.
} 
irrelevante. Nos demais, o exame da significância individual, tanto do MCE como o conjunto dos coeficientes defasados para a variável DIn(CRE), foi incapaz de rejeitar, estatisticamente, a hipótese nula, para a qual a variável explicativa não é diferente de zero. Logo, toma-se a decisão de rejeitar a hipótese de que $\operatorname{Dln}(\mathrm{CRE})$ Granger-causa Dln(RES).

Tabela 5: Estimação de Dln(CRE) por MQO

Período amostral: 1995 (1) - 1998 (12)

\begin{tabular}{|c|c|c|c|c|}
\hline Variável & Coeficiente & S.E. & $\mathbf{T}$ & HSCE \\
\hline Constante & 0,0201 & 0,012 & 1,677 & 0,016 \\
\hline$D \ln (C R E) t-1$ & $-0,4925$ & 0,191 & $-2,582$ & 0,412 \\
\hline $\operatorname{Dln}(\mathrm{CRE}) \mathrm{t}-2$ & $-0,2157$ & 0,196 & $-1,100$ & 0,479 \\
\hline $\mathrm{D} \ln (\mathrm{CRE}) \mathrm{t}-3$ & 0,0891 & 0,020 & 0,456 & 0,369 \\
\hline$D \ln (C R E) t-4$ & 0,0694 & 0,196 & 0,354 & 0,439 \\
\hline$D \ln (C R E) t-5$ & 0,1167 & 0,185 & 0,632 & 0,353 \\
\hline$D \ln (C R E) t-6$ & 0,0738 & 0,160 & 0,462 & 0,451 \\
\hline$D \ln (R E S) t-1$ & 0,0425 & 0,086 & 0,495 & 0,117 \\
\hline$D \ln ($ RES) $t-2$ & $-0,1326$ & 0,096 & $-1,385$ & 0,193 \\
\hline$D \ln (R E S) t-3$ & $-0,0999$ & 0,100 & $-0,994$ & 0,194 \\
\hline $\operatorname{DIn}(R E S) \mathrm{t}-4$ & $-0,0207$ & 0,136 & $-0,152$ & 0,183 \\
\hline$D \ln (R E S) t-5$ & 0,1737 & 0,134 & 1,298 & 0,202 \\
\hline MCE t-1 & 0,0124 & 0,066 & 0,188 & 0,132 \\
\hline $\mathrm{R}^{2}=0,362$ & $F(12,28)=1,322[0,261]$ & $S=0,042$ & $D W=2,14$ & RSS $=0,049$ \\
\hline
\end{tabular}

Teste de Causalidade de Granger

$F(5,28)=1,3474[0,2738]$

\section{Testes de Diagnóstico}

AR 1 - $4 F(4,24)=4,128[0,011]$ *

ARCH $4 F()=1,312[0,299]$

Normality Chi^2 $(2)=8,415[0,015]$ *

$X^{\wedge} 2 \quad F()=2,087[0,300]$

RESET $F()=3,467[0,073]$

${ }^{* *}$ Significativo ao nivel de $1 \% ;{ }^{*}$ Significativo ao nível de $5 \%$ 


\subsection{Dinâmica - sem inclusão do MCE}

No entanto, não obstante a execução dos procedimentos referentes a sistemas cointegrados, neste estudo, tendo em vista a análise de um curto período amostral, resolveu-se efetuar novamente o teste de causalidade de Granger, desta vez, sem a inclusão do vetor de cointegração. Como na última seção, a metodologia do teste permanece a mesma, sendo realizada em duas etapas. Assim, na primeira estimação, comparando-a com a equação 2, a disposição continua inalterada, faz-se apenas a exclusão MCE. Os resultados são apresentados na tabela 6 , abaixo.

Tabela 6: Estimação de Dln(RES) por MQO

Período amostral: 1995 (8) - 1998 (12)

\begin{tabular}{|c|c|c|c|c|}
\hline Variável & Coeficiente & S.E. & $\mathbf{T}$ & HSCE \\
\hline Constante & 0,0013 & 0,022 & 0,060 & 0,024 \\
\hline$D \ln (R E S) t-1$ & 0,3358 & 0,157 & 2,136 & 0,155 \\
\hline$D \ln (R E S) t-2$ & $-0,0358$ & 0,167 & $-0,214$ & 0,195 \\
\hline$D \ln (R E S) t-3$ & $-0,0629$ & 0,176 & $-0,356$ & 0,221 \\
\hline$D \ln (R E S) t-4$ & 0,1972 & 0,257 & 0,766 & 0,418 \\
\hline$D \ln (R E S) t-5$ & $-0,4187$ & 0,236 & $-2,067$ & 0,479 \\
\hline$D \ln (C R E) t-1$ & 0,3349 & 0,335 & 0,999 & 0,288 \\
\hline$D \ln (C R E) t-2$ & 0,2406 & 0,368 & 0,654 & 0,430 \\
\hline$D \ln (C R E) t-3$ & 0,0921 & 0,370 & 0,249 & 0,485 \\
\hline$D \ln (C R E) t-4$ & $-0,1059$ & 0,370 & $-0,286$ & 0,355 \\
\hline$D \ln (C R E) t-5$ & 0,0852 & 0,349 & 0,244 & 0,345 \\
\hline$D \ln (C R E) t-6$ & 0,1174 & 0,302 & 0,388 & 0,259 \\
\hline
\end{tabular}

$R^{2}=0,312301 \quad F(11,29)=1,1972[0,3318] \quad S=0,0179 \quad D W=2,11 \quad R S S=0,183$

\section{Teste de Causalidade de Granger}

$F(6,29)=0,25462[0,9534]$

\section{Testes de Diagnóstíco}

AR $1-4 F(4,25)=0,408[0,801]$

$\mathrm{ARCH} 4 \mathrm{~F}(4,21)=0,043[0,996]$

Normality $\mathrm{Chi}^{\wedge} 2(2)=28,271[0,000] * *$

$X_{i \wedge} 2 \quad F(22,6)=0,093[1,000]$

RESET $F(1,28)=0,069[0,795]$

*: significativo ao nivel de $1 \%$; ${ }^{*}$ significativo ao nível de $5 \%$. 
Neste primeiro exame, identificou-se pequenas mudanças, mas que, sobretudo, não são suficientes para influenciar a decisão ou a direção do teste de causalidade. Destaca-se, ainda, que, em relação aos testes estatísticos e de diagnóstico, não ocorreram alterações significativas. Comparativamente com os resultados da tabela 4, tem-se uma melhor performance do conjunto dos testes de diagnóstico, quando estes apenas acusam desvios de normalidade na distribuição dos resíduos, ao nível de $1 \%$ de significância para um valor tabelado de 22,1643 (observe o Normality teste). No mais, a incidência de um baixo coeficiente de determinação foi confirmada pelos testes t e F, que indicam estimadores com baixa eficiência, conseqüentemente, insignificantes. O teste de causalidade de Granger, como já foi destacado no início, foi efetuado tanto para o conjunto dos coeficientes defasados da variável Dln(CRE) como da Dln(RES), de acordo com os testes $\mathrm{F}$ efetuados em separado. Entretanto, nenhum deles foi capaz de provar alguma relação de precedência temporal entre as variáveis. Ou seja, rejeita-se uma possível inferência de que Dln(CRE) causa, no sentido de Granger, Dln(RES).

$\mathrm{Na}$ outra etapa, a inversão do modelo também não trouxe nenhuma indicação que desse subsídios para um posicionamento contrário. Antagonicamente, mas congruente com as demais estimações, o teste revelouse inconclusivo para indicar uma possível relação de precedência temporal, no sentido de Granger, entre as variáveis do modelo estimado (ver Tabela 7). Da mesma forma como nos outros modelos, os testes $\mathrm{F}$ e $\mathrm{t}$, para avaliar o grau de significância conjunta e individual, respectivamente, de cada variável, não demonstraram ser eficientes. Quanto aos testes de diagnóstico, repete-se a mesma observação do modelo anterior, no que diz respeito à distribuição normal dos resíduos. Entretanto, tem-se, desta vez, problemas com resíduos autocorrelacionados. As conclusões relacionadas ao teste de causalidade de Granger para a estimação em específico, não diferem da anterior, sendo insignificantes os resultados para o conjunto dos coeficientes defasados da variável $\mathrm{D} \ln (\mathrm{RES})$.

Após a realização de sucessivas estimações, como foi definido na metodologia, as observações explicitadas permitem o enquadramento em um dos casos sugeridos por Granger. Considerando que não houve divergência nos resultados apresentados com ou sem a inclusão do MCE, conclui-se por classificar os resultados em um caso de independência, tendo em vista que não ocorreu causalidade unidirecional, tampouco bidirecional ou simultaneidade. Nesta situação, o conjunto de coeficientes defasados de $D \ln (\mathrm{RES})$ e Dln(CRE), em ambas as regressões, com ou sem análise de cointegração, não é estatisticamente significante. 
Tabela 7: Estimação de Dln(CRE) por MQO

Período amostral: 1995 (8) - 1998 (12)

\begin{tabular}{l|c|c|c|c}
\hline \multicolumn{1}{c|}{ Variável } & Coeficiente & S.E. & T & HSCE \\
\hline Constante & 0,0195 & 0,011 & 1,712 & 0,011 \\
DIn(CRE) t-1 & $-0,4792$ & 0,174 & $-2,751$ & 0,356 \\
DIn(CRE) t-2 & $-0,2109$ & 0,191 & $-1,104$ & 0,459 \\
DIn(CRE) t-3 & 0,0894 & 0,192 & 0,465 & 0,348 \\
DIn(CRE) t-4 & 0,0671 & 0,192 & 0,349 & 0,434 \\
DIn(CRE) t-5 & 0,1185 & 0,181 & 0,654 & 0,344 \\
DIn(CRE) t-6 & 0,0749 & 0,157 & 0,477 & 0,449 \\
Din(RES) t-1 & 0,0384 & 0,082 & 0,470 & 0,086 \\
DIn(RES) t-2 & $-0,1395$ & 0,087 & $-1,603$ & 0,120 \\
DIn(RES) t-3 & $-0,1069$ & 0,092 & $-1,166$ & 0,170 \\
DIn(RES) t-4 & $-0,0225$ & 0,134 & $-0,168$ & 0,156 \\
Dln(RES) t-5 & 0,1646 & 0,123 & 1,343 & 0,194 \\
\hline
\end{tabular}

$R^{2}=0,361 \quad F(11,29)=1,488[0,1891] \quad S=0,041 \quad D W=2,14 \quad R S S=0,0493$

\section{Teste de Causalidade de Granger}

$F(5,29)=1,6947[0,1891]$

\section{Testes de Diagnóstico}

AR $1-4 F(4,25)=3,845[0,014]^{*}$

ARCH $4 \mathrm{~F}(4,21)=1,429[0,259]$

Normality $\mathrm{Chi}^{\wedge} 2(2)=8,736[0,012]^{*}$

$X_{i \wedge} 2 \quad F(22,6)=3,966[0,047]^{*}$

RESET $F(1,28)=2,828[0,104]$

** Significativo ao nível de $1 \%$; * Significativo ao nível de $5 \%$.

\section{Conclusão}

Os testes demonstraram que o modelo de crise de balanço de pagamento e de ataque especulativo de Krugman (1979) não explica a crise cambial brasileira deflagrada em janeiro de 1999. 
A contribuição deste trabalho consiste em rejeitar a hipótese de crise nos fundamentos como causa do ataque ao Real em janeiro de 1999. Nada indica que uma expansão do crédito doméstico tenha precedido temporalmente, ou causado, no sentido de Granger, o declínio das reservas internacionais, no período de janeiro de 1995 a dezembro de 1998.

Os resultados sugerem outras explicações para o episódio. A literatura enfatiza que o caso brasileiro parece mais condizente com os "modelos de $2^{a}$ geração", os quais explicam a crise cambial como decorrente de causas adversas em detrimento das decisões internas de política econômica (fundamentos). Para estes modelos, determinados ataques especulativos e crises cambiais podem ser explicados por profecias auto-realizáveis. Quando os agentes econômicos antecipam que o custo para a manutenção do regime cambial fixo é maior que o seu beneficio, e o policymaker também percebe que o custo de uma defesa da paridade cambial (ataque especulativo) é maior que o custo para abandonar o regime cambial, e dado que o mercado sabe dessas informações, então a profecia da mudança do regime cambial realiza-se.

Aliado a esse pensamento, no caso brasileiro, a literatura enfatiza que a crise cambial em estudo deve-se a problemas de credibilidade das políticas econômicas adotadas durante o Plano Real. A idéia é que a política não era crível, pois os custos para sua manutenção foram muito elevados, com altas taxas de desemprego e um forte crescimento da dívida pública. Para um melhor entendimento ver Drazen e Masson (1994), Giambiagi e Moreira (1999), Arbex e Fontes (1999).

\section{Referências bibliográficas}

ALENCAR, Leonardo Soriano de. "Raízes unitárias e cointegração: uma introdução”. Boletim do Banco Central do Brasil, p.171-206, Abr. 1998. ARBEX, Marcelo A. e FONTES, Rosa. "Credibilidade das Políticas Econômicas no Brasil: Uma Análise Empírica do Período 1991-1998". Economia Aplicada, V. 3, n.1, 1999.

CARNEIRO, F. G. A Metodologia dos Testes de Causalidade em Economia. Brasília: Editora da Unb. (Série textos didáticos n. ${ }^{\circ} 20$ ), p.1-19, 1997.

CHAREMZA, Wojciech W. e DEADMAN, Derek F. "New Directions" in Econometric Practice. Second Edition, Edward Elgar Publishing Limited, 1997. 
DIVINO, José Angelo C. A. Agregação Monetária Ponderada: uma Análise de Causalidade. XXVI Encontro Nacional de Economia. p. 705-725, 1998.

DRAZEN, A. e MASSON, P. "Credibility of Policies Versus Credibility of Policy Makers". The Quarterly Journal of Economics, p. 735-754, Aug. 1994.

ENDERS, W. Applied econometric time series. $1^{\text {a }}$ edition. Iowa: John Wiley E Sons Inc., 1995.

ENGLE, R. F. e GRANGER, C. W.J. "Co-intergration and Error Correrction: Representation, Estimation, and Testing". Econometrica, n.55, p. 251-276, 1987.

FLOOD e GARBER. "Collapsing Exchange-Rate Regimes: Some Linear Examples", Journal of International Economics, n.17, p. 1- 13, 1984.

GIAMBIAGI, Fábio e MOREIRA, Maurício Mesquita. "Taxas de Juros e de Câmbio Real após a Desvalorização do Real: Um Cenário Tentativo para 1999/2002". Revista do BNDES, Junho, 1999.

GRANGER, C. W. J. "Investigating Causal Relations by Econometric Models and a Cross-Spectral Models". Econometrica, n. 34, p. 541-51, 1969.

HERMANN, Jennifer. "Ancoragem Cambial em Ambiente de Elevada Mobilidade Internacional do Capital: Alcance. Limites e Soluções". Estudos Econômicos, USP, São Paulo, n. 29(v. 4), p. 475-511, out./dez., 1999.

JOHANSEN, S. "Statistical analysis of cointegration vectors". Journal of Economic Dynamics and Control 12, p. 231-54, 1988.

KRUGMAN, Paul. "A Model of Balance-of-Payments Crises". Journal of Money, Credit, and Banking, (11), p. 311-25, n. 3, August, 1979.

MADDALA, G. S. e KIM, In-Moo. Unit Roots, Cointegration, and Structural Change. Cambridge University Press, 2000.

MIRANDA, Mauro Costa. Crises Cambiais e Ataques Especulativos no Brasil: janeiro de 1982 a janeiro de 1999. UNB. Jul., 1999.

MOLLO, Maria de Lourdes Rollemberg e SILVA, Maria Luiza Falcão. "A Liberalização do Câmbio no Brasil: Revisitando a Discussão dos Pressupostos Teóricos Embutidos nas Prescrições Cambiais Alternativas”. Estudos Econômicos, USP, São Paulo, n.29(2), p.189-227, abr.jun., 1999. 
OBSTFELD, M. The Logic of Currency Crises. (Working Paper n. 4, p. 640).Cambridge: National Bureau of Economic Research, 1994.

SCHWARTSMAN, Alexandre. "A crise cambial e o ajuste fiscal". Revista de Economia Política, (19), n. ${ }^{\circ}$ 1, p. 73, jan-mar, 1999. 